\title{
ON GROVER'S SEARCH ALGORITHM FROM A QUANTUM INFORMATION GEOMETRY VIEWPOINT
}

\author{
Carlo Cafaro ${ }^{1}$ and Stefano Mancini ${ }^{2}$ \\ ${ }^{1,2}$ School of Science and Technology, Physics Division, \\ University of Camerino, I-62032 Camerino, Italy
}

\begin{abstract}
We present an information geometric characterization of Grover's quantum search algorithm. First, we quantify the notion of quantum distinguishability between parametric density operators by means of the Wigner-Yanase quantum information metric. We then show that the quantum searching problem can be recast in an information geometric framework where Grover's dynamics is characterized by a geodesic on the manifold of the parametric density operators of pure quantum states constructed from the continuous approximation of the parametric quantum output state in Grover's algorithm. We also discuss possible deviations from Grover's algorithm within this quantum information geometric setting.

PACS numbers: Probability Theory (02.50.Cw); Quantum Algorithms (03.67.Ac), Riemannian Geometry (02.40.Ky).
\end{abstract}

\section{INTRODUCTION}

Information geometry is the application of differential geometric techniques to the study of families of probabilities, both classical and quantum, either parametric or nonparametric [1]. Much of the work in its quantum version has been concentrated on manifolds of density operators for both finite and infinite dimensional quantum systems. Classical and quantum Fisher information metrics on statistical manifolds play a key-role in information geometry, especially in the geometric and informational approach to classical and quantum estimation theory, respectively [1]. Applications of information geometric techniques appear in statistical mechanics, in the study of the three-dimensional spherical model [2, 3], the Ising model on planar random graphs [ㅍ, 4] and the one-dimensional Potts model [3, 5]. Furthermore, the Fisher information plays a fundamental role in quantifying Heisenberg's uncertainty principle from a statistical inference point of view [6]. Indeed, information geometric methods in combination with probable inference techniques have been recently employed to define the notion of distinguishability between probability distributions of curved statistical manifolds underlying the information dynamics used to provide a probabilistic description of physical systems when only partial knowledge (incomplete information) about them is available 7 - 10] . This line of investigation is very stimulating and appealing, especially considering that it is an old dream that of viewing quantum mechanics as rooted in making statistical inferences based on observed experimental data [11, 12]. Recent works where information geometry and inference methods are used to investigate the origin of fundamental theories as information geometric inferential theories appear in $[13-15]$.

Very recently, we have observed that information theory has motivated conventional differential geometric investigations of quantum state spaces. For instance, Riemannian geometric tools have been used to characterize the quantum gate complexity in quantum computing [16 18]. In [16], the problem of finding optimal quantum circuits was recast as a differential geometric problem. Specifically, a Riemannian metric on a manifold of multi-qubit unitary transformations is used to define a metric distance between the identity operator and the desired unitary operator representing the quantum computation. The metric distance turns out to be equivalent to the number of quantum gates needed to represent that unitary operator. It was then shown that finding optimal quantum circuits is essentially equivalent to finding the shortest path (geodesic) between two points in a certain curved geometry. However, the number of investigations concerning the applications of quantum information geometric methods to specific problems of quantum computing, such as quantum estimation, quantum channel entropy, quantum noise and entanglement and quantum algorithms is very limited. This is not unreasonable considering that the systematic study of quantum information geometry has only started no more than ten years ago [1].

In this paper, we provide a simple application of quantum information geometry to quantum computing. It is known that Grover's quantum search algorithm [19] can be viewed as a geodesic path on the manifold of Hilbert-space rays where the notion of quantum distinguishability is quantified via the Fubini-Study metric, a gauge invariant metric on the projective Hilbert space [20, 21]. By observing that a parametric quantum wavefunction induces in a natural manner a parametric density operator and by considering its square root, we use the concept of Wigner-Yanase quantum information metric. Such metric is one among many versions of a so-called quantum Fisher information metric, a metric on manifolds of density operators for both finite and infinite dimensional quantum systems. We show in an explicit manner that the Wigner-Yanase metric and the Fubini-Study metric differ by a factor of four when considering pure state models. Finally, interpreting the Fubini-Study metric as a quantum version of Fisher 
metric, we provide an information geometric characterization of Grover's algorithm as a geodesic (shortest length curve) in the parameter space characterizing the pure state model, the manifold of the parametric density operators of pure quantum states. Finally, we discuss few possible deviations (non-constant Fisher information function and/or non-actuality) from Grover's algorithm within this quantum information geometric framework.

The layout of the article is as follows. In Section II, we introduce the Wigner-Yanase quantum information metric from both formal and heuristic viewpoints. In Section III, we briefly discuss Grover's search algorithm in quantum computing. In Section IV, we show that Grover's dynamics corresponds to the shortest path from an information geometric point of view. In Section V, we consider deviations from Grover's probability path where the requirements of constant Fisher information function and/or actuality are not simultaneously satisfied. Our concluding remarks appear in Section VI.

\section{ON INFORMATION GEOMETRY AND STATISTICAL DISTINGUISHABILITY}

In the classical setting, it is known that except for an overall multiplicative constant the classical Fisher information metric is unique [22, 23]: it is the only monotone Riemannian metric with the property of having its line element reduced under Markov morphisms (stochastic maps). Said otherwise, there is essentially one classical statistical distance quantifying the classical distinguishability between two probability distributions. In the quantum setting, Riemannian metrics are considered on the space of density matrices. The requirement that the distance between density matrices expresses quantum statistical distinguishability implies that this distance must decrease under coarsegraining (stochastic maps). Unlike the classical case, it turns out that there are infinitely many monotone Riemannian metrics satisfying this requirement [24 26].

In this section we introduce the Wigner-Yanase quantum information metric from both a formal and heuristic point of view.

\section{A. The Wigner-Yanase Quantum Information Metric: the Formal Approach}

Assume that $\mathcal{M}_{n}$ denotes the space of $(n \times n)$-complex matrices, $\mathcal{D}_{n}$ is the manifold of strictly positive elements of $\mathcal{M}_{n}$ and $\mathcal{D}_{n}^{1} \subset \mathcal{D}_{n}$ is the submanifold of density matrices,

$$
\mathcal{D}_{n}^{1} \stackrel{\text { def }}{=}\left\{D \in \mathcal{D}_{n}: D=D^{\dagger} \text { and } \operatorname{Tr}(D)=1\right\}
$$

The tangent space to $\mathcal{D}_{n}^{1}$ at $\rho$ is given by,

$$
T_{\rho} \mathcal{D}_{n}^{1} \stackrel{\text { def }}{=}\left\{D \in \mathcal{M}_{n}: D=D^{\dagger} \text { and } \operatorname{Tr}(D)=0\right\} .
$$

Given $D_{1}$ and $D_{2}$ in $T_{\rho} \mathcal{D}_{n}^{1}$, the Wigner-Yanase quantum information metric is defined as [27],

$$
\left\langle D_{1}, D_{2}\right\rangle_{\rho, f_{\mathrm{WY}}} \stackrel{\text { def }}{=} \operatorname{Tr}\left[D_{1} \cdot c_{\mathrm{WY}}\left(L_{\rho}, R_{\rho}\right)\left(D_{2}\right)\right]
$$

where $L_{\rho}$ and $R_{\rho}$ are the left and right multiplication operators, respectively, with

$$
L_{\rho}(D) \stackrel{\text { def }}{=} \rho D \text { and, } R_{\rho}(D) \stackrel{\text { def }}{=} D \rho .
$$

The quantity $c_{\mathrm{WY}}\left(L_{\rho}, R_{\rho}\right)$ in (3) denotes the so-called Chentsov-Morozova function for the Wigner-Yanase information metric,

$$
c_{\mathrm{WY}}(x, y) \stackrel{\text { def }}{=} \frac{1}{y f_{\mathrm{WY}}\left(\frac{x}{y}\right)}=\frac{4}{(\sqrt{x}+\sqrt{y})^{2}} \text { with } x, y>0 \text {. }
$$

The function $f:(0,+\infty) \rightarrow \mathbb{R}$ is an operator monotone function, that is $\forall n \in \mathbb{N}$ and $\forall M_{1}, M_{2} \in \mathcal{M}_{n}$ with $0 \leq M_{1} \leq M_{2}$, it must be $0 \leq f\left(M_{1}\right) \leq f\left(M_{2}\right)$. To evaluate $\left\langle D_{1}, D_{2}\right\rangle_{\rho, f_{\mathrm{WY}}}$ in (3), observe that $T_{\rho} \mathcal{D}_{n}^{1}$ can be decomposed in two orthogonal subspaces $\left(T_{\rho} \mathcal{D}_{n}^{1}\right)^{\mathrm{c}}$ and $\left(T_{\rho} \mathcal{D}_{n}^{1}\right)^{\mathrm{O}}$,

$$
T_{\rho} \mathcal{D}_{n}^{1}=\left(T_{\rho} \mathcal{D}_{n}^{1}\right)^{\mathrm{c}} \oplus\left(T_{\rho} \mathcal{D}_{n}^{1}\right)^{\mathrm{o}}
$$


where,

$$
\left(T_{\rho} \mathcal{D}_{n}^{1}\right)^{\mathrm{c}}=\left\{D \in T_{\rho} \mathcal{D}_{n}^{1}:[D, \rho]=0\right\}
$$

and $\left(T_{\rho} \mathcal{D}_{n}^{1}\right)^{\mathrm{o}}$ is the orthogonal complement of $\left(T_{\rho} \mathcal{D}_{n}^{1}\right)^{\mathrm{c}}$. Therefore, an arbitrary element $D^{\prime}$ of $\left(T_{\rho} \mathcal{D}_{n}^{1}\right)^{\mathrm{o}}$ can be written as $i[\rho, D]$ with $D=D^{\dagger}$. Thus,

$$
\left\langle D^{\prime}, D^{\prime}\right\rangle_{\rho, f_{\mathrm{WY}}}=\langle i[\rho, D], i[\rho, D]\rangle_{\rho, f_{\mathrm{WY}}}=\operatorname{Tr}\left\{i[\rho, D] 4\left(L_{\rho}^{\frac{1}{2}}+R_{\rho}^{\frac{1}{2}}\right)^{-2}(i[\rho, D])\right\}=-4 \operatorname{Tr}\left(\left[\rho^{\frac{1}{2}}, D\right]^{2}\right),
$$

where we have made use of the following two relations,

$$
[\rho, D]=\left(L_{\rho}-R_{\rho}\right)(D)=\left(L_{\rho}^{\frac{1}{2}}+R_{\rho}^{\frac{1}{2}}\right)\left(L_{\rho}^{\frac{1}{2}}-R_{\rho}^{\frac{1}{2}}\right)(D)
$$

and,

$$
L_{\rho}^{\frac{1}{2}}(D)=\rho^{\frac{1}{2}} D, R^{\frac{1}{2}}(D)=D \rho^{\frac{1}{2}} .
$$

We notice that $\left\langle D^{\prime}, D^{\prime}\right\rangle_{\rho, f_{\mathrm{WY}}}$ in (8) can be written as,

$$
I_{\mathrm{WY}}(\rho) \equiv\left\langle D^{\prime}, D^{\prime}\right\rangle_{\rho, f_{\mathrm{WY}}}=8 I(\rho, D),
$$

where $I(\rho, D)$ defined as,

$$
I(\rho, D) \stackrel{\text { def }}{=}-\frac{1}{2} \operatorname{Tr}\left(\left[\rho^{\frac{1}{2}}, D\right]^{2}\right),
$$

is the skew information introduced by Wigner and Yanase when studying quantum measurement theory from an information-theoretic viewpoint [28].

\section{B. The Wigner-Yanase Quantum Information Metric: the Heuristic Approach}

The classical Fisher information $I_{F}\left(p_{\theta}\right)$ of a parametric probability density $p_{\theta}(x)$ is a key-quantity in the statistical estimation theory and it is defined as,

$$
I_{F}\left(p_{\theta}\right) \stackrel{\text { def }}{=} \int d x p_{\theta}(x)\left(\frac{\partial \log p_{\theta}(x)}{\partial \theta}\right)^{2} .
$$

Observe that $I_{F}\left(p_{\theta}\right)$ can be rewritten in terms of a fundamental quantity in quantum physics, the probability amplitude (wavefunction) $\sqrt{p_{\theta}(x)}$,

$$
I_{F}\left(p_{\theta}\right)=4 \int d x\left(\frac{\partial \sqrt{p_{\theta}(x)}}{\partial \theta}\right)^{2} .
$$

When going from classical to quantum theory, a quantum version of the classical Fisher information can be heuristically defined as follows: the integral in (14) is replaced by the trace and the probability densities $p_{\theta}$ by density operators $\rho_{\theta}$. Therefore $I_{F}\left(\rho_{\theta}\right)$, a quantum extension of $I_{F}\left(p_{\theta}\right)$ to a family of parametric quantum operators $\rho_{\theta}$, reads

$$
I_{F}\left(\rho_{\theta}\right)=4 \operatorname{Tr}\left(\frac{\partial \sqrt{\rho_{\theta}}}{\partial \theta}\right)^{2} .
$$

Quantum generalizations of the very same classical Fisher information are not unique. Indeed, two classically identical expressions (see (13) and (14), for instance) generally differ when they are extended to a quantum setting. This difference is a manifestation of the non-commutative nature of quantum mechanics and is reminiscent of the idea of quantum discord [29].

If $\rho_{\theta}$ in (15) satisfies the von Neumann equation,

$$
\frac{\partial \rho_{\theta}}{\partial \theta}+i\left[T, \rho_{\theta}\right]=0
$$


with $\rho_{0}=\rho, \theta \in \mathbb{R}$ is a temporal parameter and $T$ is the generator of temporal shift. Then,

$$
\rho_{\theta}=e^{-i \theta T} \rho e^{i \theta T} \text { and, } \frac{\partial \sqrt{\rho_{\theta}}}{\partial \theta}=i e^{-i \theta T}\left[\rho^{\frac{1}{2}}, T\right] e^{i \theta T} .
$$

Substituting the second equation in (17) into (15), $I_{F}\left(\rho_{\theta}\right)$ becomes independent of $\theta$ and reads

$$
I_{F}\left(\rho_{\theta}\right)=8 I(\rho, T),
$$

where $I(\rho, T)$ is the Wigner-Yanase skew information defined in (12). Thus, the skew information is a particular kind of quantum Fisher information $I_{F}\left(\rho_{\theta}\right)$ known as the Wigner-Yanase quantum Fisher information

$$
I_{\mathrm{WY}}\left(\rho_{\theta}\right) \equiv I_{F}\left(\rho_{\theta}\right)=8 I(\rho, T) .
$$

When a density operator is parametrized by $n$-real parameters $\theta \equiv\left(\theta^{1}, \ldots, \theta^{n}\right)$ and the quantum state $\rho_{\theta}$ satisfies the von Neumann equation, the set of quantum states $\rho_{\theta}$ forms the quantum evolution submanifold of $\mathcal{D}_{n}^{1}$. The set of parameters $\theta$ can be regarded as a local coordinate system on such submanifold endowed with a metric structure defined by the information metric $g_{i j}^{(\mathrm{WY})}(\theta)$,

$$
g_{i j}^{(\mathrm{WY})}(\theta)=4 \operatorname{Tr}\left[\left(\partial_{i} \sqrt{\rho_{\theta}}\right)\left(\partial_{j} \sqrt{\rho_{\theta}}\right)\right], \partial_{j} \equiv \frac{\partial}{\partial \theta^{j}} .
$$

Once the monotone Riemannian metric $g_{i j}^{(\mathrm{WY})}(\theta)$ is explicitly obtained, the other geometric quantities (Christoffel connection coefficients, scalar and sectional curvatures, Riemannian curvature tensor, etc.) can be calculated from it in a purely mathematical way, at least in principle. For instance, the geodesic equation reads [30],

$$
D \dot{\theta}^{k} \stackrel{\text { def }}{=}\left(\frac{\partial \dot{\theta}^{k}}{\partial \theta^{j}}+\Gamma_{i j}^{k}(\theta) \dot{\theta}^{i}\right) d \theta^{j}=\frac{d^{2} \theta^{k}(\tau)}{d \tau^{2}}+\Gamma_{i j}^{k}(\theta) \frac{d \theta^{i}}{d \tau} \frac{d \theta^{j}}{d \tau}=0
$$

where $D$ denotes the covariant derivative, $\dot{\theta}^{k} \stackrel{\text { def }}{=} \frac{d \theta^{k}}{d \tau}$ and,

$$
\Gamma_{i j}^{k}(\theta) \stackrel{\text { def }}{=} \frac{1}{2} g^{k l}\left(\partial_{i} g_{l j}+\partial_{j} g_{i l}-\partial_{l} g_{i j}\right),
$$

are the Christoffel connection coefficients.

\section{ON GROVER'S SEARCH ALGORITHM}

In this section we briefly discuss what is considered a masterpiece of quantum computational software [31], that is Grover's search algorithm in quantum computing.

\section{A. Quantum Searching}

The search problem may be stated as follows: we wish to retrieve a certain item satisfying a given condition assuming that it belongs to an unsorted database (oracle) containing $N=2^{n}$ items. One step is needed to specify whether or not the examined item is the one fulfilling the given condition. We assume that the selection of the item is aided by no sorting on the database. In such a situation, the most efficient classical algorithm to tackle the search problem requires the examination of the items in the database one by one. Therefore, by means of a classical computer, the oracle must be queried on average $\frac{N}{2}$ times ( $O(N)$ classical steps). However, by using the same amount of hardware as in the classical case but by having the input and output in superpositions of states, Grover has developed a quantum mechanical algorithm capable of solving this search problem in about $\frac{\pi}{4} \sqrt{N}$ steps $(O(\sqrt{N})$ quantum mechanical steps) [19]. Although this is not dramatic as the exponential quantum advantage achieved by Shor's algorithm for factoring, the extremely wide applicability of searching problems makes Grover's algorithm interesting and important. In particular, Grover's algorithm gives a quadratic speed-up in the solution of $N P$-complete problems, which account for many of the important hard problems in computer science. Indeed, it was shown in 32] that relative to an oracle chosen uniformly at random, with probability 1, the class NP cannot be solved on a quantum Turing machine in time 
$O(\sqrt{N})$. Drawing on this result, Grover pointed out in [33] that his algorithm is optimal (i.e., the fastest), up to a multiplicative constant factor, among all possible quantum algorithms. A detailed proof of Grover's statement about the optimality of his quantum searching algorithm appears in [34]. Finally, in [35], it is shown that for any number of oracle lookups, Grover's algorithm is exactly (and not just asymptotically) optimal.

What makes a quantum search algorithm more efficient than another? An algorithm is an abstract mathematical concept, whereas it is useful to consider how efficiently we can run an algorithm on a computer. Computer scientists associate a cost with each step of the algorithm and with the amount of memory required, embodying the idea that physical computers have a finite size (memory) and work at a finite rate of elementary calculation steps per unit time. This gives us a way to determine if one algorithm is intrinsically faster than another. In general, it is very difficult to formally prove that a certain algorithm is the "best" algorithm for a given computational task. For instance, Shor's factoring algorithm [36] is only the "best known" algorithm for factoring; there is no proof that something faster cannot be found in the future.

\section{B. Grover's Algorithm}

In what follows, we outline the construction of Grover's algorithm when considering the $n$-qubit case $\left(N=2^{n}\right.$ states). The step-0 (the initialization) of Grover's algorithm begins by using the Hadamard transform to construct a uniform amplitude initial state which is an equal superposition of all the orthonormal computational basis states $\{|s\rangle\}$ in the $N$-dimensional Hilbert space,

$$
|\bar{q}\rangle=\frac{1}{\sqrt{N}} \sum_{s=0}^{N-1}|s\rangle
$$

Observe that the state (23) may be rewritten as,

$$
|\bar{q}\rangle=\frac{1}{\sqrt{N}} \sum_{s=0}^{N-1}|s\rangle=\sqrt{\frac{N-1}{N}} \sqrt{\frac{1}{N-1}} \sum_{s \neq a}|s\rangle+\frac{1}{\sqrt{N}}|a\rangle=\cos \left(\frac{\alpha}{2}\right)|r\rangle+\sin \left(\frac{\alpha}{2}\right)|a\rangle,
$$

where the angle $\alpha$ characterizes the overlap between $|\bar{q}\rangle$ and the searched state $|a\rangle$,

$$
\sin \left(\frac{\alpha}{2}\right) \stackrel{\text { def }}{=} \frac{1}{\sqrt{N}} \equiv\langle a \mid \bar{q}\rangle
$$

while the state $|r\rangle$ is defined as,

$$
|r\rangle \stackrel{\text { def }}{=} \sqrt{\frac{1}{N-1}} \sum_{s \neq a}|s\rangle
$$

Given the initial input, a single-step of Grover's algorithm is characterized by a rotation by $\alpha$ in the two-dimensional space spanned by $|r\rangle$ and $|a\rangle$. The effect of the algorithm after $m$-steps leads to the state $\left|\psi_{\mathrm{G}}(m)\right\rangle$,

$$
\left|\psi_{\mathrm{G}}(m)\right\rangle=\cos \left[\left(m+\frac{1}{2}\right) \alpha\right]|r\rangle+\sin \left[\left(m+\frac{1}{2}\right) \alpha\right]|a\rangle .
$$

In the limit of $N \gg 1$, the number of steps $\bar{m}$ for which $\left|\psi_{\mathrm{G}}(\bar{m})\right\rangle$ coincides with the searched state $|a\rangle$ (with success probability equal to 1 ) is approximately given by,

$$
\bar{m} \stackrel{N \geqq 1}{\simeq} \frac{\pi}{4} \sqrt{N}
$$

Equation (28) can be obtained by imposing that $\left(\bar{m}+\frac{1}{2}\right) \alpha=\frac{\pi}{2}$ and by observing that when $N \gg 1$, Eq. (25) implies that $\alpha \simeq \frac{2}{\sqrt{N}}$.

Although Grover's algorithm evolves with discrete $m$, in the limit of $N \gg 1$ the output state (27) can be approximated by a quantum wave-state $|\psi(\theta)\rangle$ depending on a continuous parameter $\theta$. Indeed, considering the following formal substitutions,

$$
\left(m+\frac{1}{2}\right) \alpha \rightarrow \theta,|a\rangle \rightarrow|0\rangle,|r\rangle \rightarrow \frac{1}{\sqrt{N-1}} \sum_{k=1}^{N-1}|k\rangle
$$


the state $|\psi(\theta)\rangle$ reads,

$$
|\psi(\theta)\rangle=\sum_{k=0}^{N-1} \sqrt{p_{k}(\theta)}|k\rangle
$$

where,

$$
\left\langle k \mid k^{\prime}\right\rangle=\delta_{k k^{\prime}}, p_{0}(\theta) \stackrel{\text { def }}{=} \sin ^{2} \theta \text { and, } p_{j}(\theta) \stackrel{\text { def }}{=} \frac{\cos ^{2} \theta}{N-1} \text { with } j \neq 0 .
$$

The $N$-dimensional probability distribution vector $\vec{p} \equiv\left(p_{0}(\theta), p_{1}(\theta), \ldots, p_{N-1}(\theta)\right)$ with $p_{k}(\theta)$ defined in (31) can be regarded as a path characterizing Grover's algorithm on a suitable probability space. In what follows, we show that such a path is indeed a geodesic path for which the quantum Fisher information action functional achieves an extremal value.

\section{INFORMATION GEOMETRY AND GROVER'S ALGORITHM}

In this section, we show that Grover's dynamics leading to (30) corresponds to the shortest path from an information geometric point of view.

\section{A. The Parametric Pure State Model}

Consider the parametric density operator $\rho_{\theta}$ constructed using the quantum state $|\psi(\theta)\rangle \equiv\left|\psi_{\theta}\right\rangle$ in (30),

$$
\rho_{\theta} \stackrel{\text { def }}{=}\left|\psi_{\theta}\right\rangle\left\langle\psi_{\theta}\left|=\sum_{k, j=0}^{N-1}\left[p_{k}(\theta) p_{j}(\theta)\right]^{\frac{1}{2}} e^{-i\left[\phi_{j}(\theta)-\phi_{k}(\theta)\right]}\right| k\right\rangle\langle j|,
$$

where the pure state $\left|\psi_{\theta}\right\rangle$ is normalized to one. Since $\rho_{\theta}$ is a pure state, it satisfies the following relations,

$$
\rho_{\theta}^{2}=\rho_{\theta} \text { and, } \rho_{\theta}=\sqrt{\rho_{\theta}} .
$$

We assume that the notion of distinguishability on the manifold of density operators defined in (32) is provided by the Wigner-Yanase quantum Fisher metric defined as,

$$
\left[I_{W Y}^{\text {(quantum) }}\right]_{i j}\left(\rho_{\theta}\right) \stackrel{\text { def }}{=} 4 \operatorname{Tr}\left[\left(\partial_{i} \sqrt{\rho_{\theta}}\right)\left(\partial_{j} \sqrt{\rho_{\theta}}\right)\right]=4 \operatorname{Tr}\left[\left(\partial_{i} \rho_{\theta}\right)\left(\partial_{j} \rho_{\theta}\right)\right]
$$

where in the multi-parametric case $\theta \equiv\left(\theta^{1}, \ldots, \theta^{l}\right)$ and $\partial_{i} \stackrel{\text { def }}{=} \frac{\partial}{\partial \theta^{i}}$ with $i=1, \ldots, l$. The second equality in (34) holds because $\rho_{\theta}$ is a pure state in the case being considered. After some algebraic manipulations and observing that the normalization condition for $\left|\psi_{\theta}\right\rangle$ implies that,

$$
\left\langle\partial_{j} \psi_{\theta} \mid \psi_{\theta}\right\rangle=-\left\langle\psi_{\theta} \mid \partial_{j} \psi_{\theta}\right\rangle
$$

the Wigner-Yanase quantum Fisher metric becomes,

$$
\left[I_{W Y}^{\text {(quantum) }}\right]_{i j}\left(\rho_{\theta}\right)=4\left[\left\langle\partial_{i} \psi_{\theta} \mid \partial_{j} \psi_{\theta}\right\rangle+\left\langle\partial_{i} \psi_{\theta} \mid \psi_{\theta}\right\rangle\left\langle\partial_{j} \psi_{\theta} \mid \psi_{\theta}\right\rangle\right] .
$$

Thus, the infinitesimal line element on the manifold of density operators in (32) reads,

$$
d s_{\mathrm{WY}}^{2}=\left[I_{W Y}^{\text {(quantum })}\right]_{i j}\left(\rho_{\theta}\right) d \theta_{i} d \theta_{j}=4\left[\left\langle\partial_{i} \psi_{\theta} \mid \partial_{j} \psi_{\theta}\right\rangle+\left\langle\partial_{i} \psi_{\theta} \mid \psi_{\theta}\right\rangle\left\langle\partial_{j} \psi_{\theta} \mid \psi_{\theta}\right\rangle\right] d \theta_{i} d \theta_{j}
$$

Defining $\gamma_{i j}$ and $\sigma_{i j}$ as,

$$
\gamma_{i j} \stackrel{\text { def }}{=} \operatorname{Re}\left[\left\langle\partial_{i} \psi_{\theta} \mid \partial_{j} \psi_{\theta}\right\rangle\right] \text { and } \sigma_{i j} \stackrel{\text { def }}{=} \operatorname{Im}\left[\left\langle\partial_{i} \psi_{\theta} \mid \partial_{j} \psi_{\theta}\right\rangle\right]
$$


respectively, it follows that

$$
\gamma_{i j}=\gamma_{j i} \text { and, } \sigma_{i j}=-\sigma_{j i}
$$

Because of the asymmetry of $\sigma_{i j}$, we have

$$
\sigma_{i j} d \theta_{i} d \theta_{j}=-\sigma_{j i} d \theta_{i} d \theta_{j} \equiv-\sigma_{j i} d \theta_{j} d \theta_{i}=-\sigma_{i j} d \theta_{i} d \theta_{j},
$$

that is,

$$
\sigma_{i j} d \theta_{i} d \theta_{j}=0
$$

Therefore, it turns out that $d s_{\mathrm{WY}}^{2}$ in (37) becomes

$$
d s_{\mathrm{WY}}^{2}=\left[I_{W Y}^{\text {(quantum) }}\right]_{i j}\left(\rho_{\theta}\right) d \theta_{i} d \theta_{j}=4\left[\operatorname{Re}\left\langle\partial_{i} \psi_{\theta} \mid \partial_{j} \psi_{\theta}\right\rangle+\left\langle\partial_{i} \psi_{\theta} \mid \psi_{\theta}\right\rangle\left\langle\partial_{j} \psi_{\theta} \mid \psi_{\theta}\right\rangle\right] d \theta_{i} d \theta_{j}
$$

We remark that $d s_{\mathrm{WY}}^{2}$ in (42) is exactly four times the Fubini-Study infinitesimal line element is given by [37],

$$
d s_{\mathrm{FS}}^{2}=g_{i j}^{(\mathrm{FS})}(\theta) d \theta_{i} d \theta_{j}=\|d \psi\|^{2}-|\langle\psi \mid d \psi\rangle|^{2}=1-\left|\left\langle\psi^{\prime} \mid \psi\right\rangle\right|^{2}
$$

where $|\psi\rangle=|\psi(\theta)\rangle \equiv\left|\psi_{\theta}\right\rangle,\left|\psi^{\prime}\right\rangle=|\psi(\theta+d \theta)\rangle \equiv\left|\psi_{\theta+d \theta}\right\rangle$ and the Fubini-Study metric $g_{i j}^{(\mathrm{FS})}(\theta)$ reads,

$$
g_{i j}^{(\mathrm{FS})}(\theta) \equiv g_{i j}(\theta) \stackrel{\text { def }}{=}\left[\operatorname{Re}\left\langle\partial_{i} \psi_{\theta} \mid \partial_{j} \psi_{\theta}\right\rangle+\left\langle\partial_{i} \psi_{\theta} \mid \psi_{\theta}\right\rangle\left\langle\partial_{j} \psi_{\theta} \mid \psi_{\theta}\right\rangle\right]
$$

The Fubini-Study metric is a gauge invariant metric on the manifold of Hilbert-space rays (projective Hilbert space). Our analysis explicitly recognizes that the Fubini-Study metric is a quantum version of the Fisher metric [38].

\section{B. Grover's Information Geometric Dynamics}

Substituting (43) into (42) and using (30) together with the normalization condition on the probabilities in (31), after some straightforward algebra it turns out that the infinitesimal Wigner-Yanase line element reads (for further details, see Appendix A),

$$
d s_{\mathrm{WY}}^{2}=\left\{\sum_{k=0}^{N-1} \frac{\dot{p}_{k}^{2}}{p_{k}}+4\left[\sum_{k=0}^{N-1} p_{k} \dot{\phi}_{k}^{2}-\left(\sum_{k=0}^{N-1} p_{k} \dot{\phi}_{k}\right)^{2}\right]\right\} d \theta^{2}
$$

where,

$$
\dot{p}_{k} \stackrel{\text { def }}{=} \frac{d p_{k}(\theta)}{d \theta} \text { and, } \dot{\phi}_{k} \stackrel{\text { def }}{=} \frac{d \phi_{k}(\theta)}{d \theta} .
$$

Recall that Grover's algorithm consists only of a sequence of unitary transformations on a pure state. In the continuous approximation, such sequence of quantum unitary operations leads to the output state $|\psi(\theta)\rangle$ in (30). Such quantum mechanical wave-vector is such that its Fisher information function $\mathcal{F}(\theta)$ [39] is independent of the parameter $\theta$ (it is a constant quantity),

$$
\mathcal{F}(\theta) \stackrel{\text { def }}{=} \sum_{k=0}^{N-1} p_{k}\left(\frac{\partial \log p_{k}}{\partial \theta}\right)^{2}=\sum_{k=0}^{N-1} \frac{\dot{p}_{k}^{2}}{p_{k}}=4 \sum_{k=0}^{N-1}\left(\frac{\partial \sqrt{p_{k}}}{\partial \theta}\right)^{2}=4 .
$$

We point out that the general expression of the Fisher information function $\mathcal{F}(\theta)$ in (47) is invariant under unitary transformations. This can be explained as follows. Consider a normalized pure state $|\psi(\theta)\rangle$ given by,

$$
|\psi(\theta)\rangle \stackrel{\text { def }}{=} \sum_{k=0}^{N-1} \sqrt{p_{k}(\theta)} e^{i \phi_{k}(\theta)}|k\rangle .
$$

Eq. (48) implies that,

$$
\left|\psi_{\theta}(m)\right|=\sqrt{p_{m}(\theta)}
$$


where $\psi_{\theta}(m) \stackrel{\text { def }}{=}\langle m \mid \psi(\theta)\rangle$ with $\langle m \mid k\rangle=\delta_{m k}$ and the Fisher information function reads,

$$
\mathcal{F}(\theta)=4 \sum_{m=0}^{N-1}\left(\frac{\partial\left|\psi_{\theta}(m)\right|}{\partial \theta}\right)^{2}=\sum_{m=0}^{N-1} \frac{\dot{p}_{m}^{2}}{p_{m}} .
$$

Under unitary transformations $U$ mapping $|\psi(\theta)\rangle$ to $\left|\psi^{\prime}(\theta)\right\rangle=U|\psi(\theta)\rangle$, the transformed Fisher information function $\mathcal{F}^{\prime}(\theta)$ becomes,

$$
\mathcal{F}^{\prime}(\theta)=4 \sum_{m=0}^{N-1}\left(\frac{\partial\left|\psi_{\theta}^{\prime}\left(u_{m}\right)\right|}{\partial \theta}\right)^{2}=\sum_{m=0}^{N-1} \frac{\dot{p}_{m}^{2}}{p_{m}}
$$

where $\psi_{\theta}^{\prime}\left(u_{m}\right) \stackrel{\text { def }}{=}\left\langle u_{m} \mid \psi^{\prime}(\theta)\right\rangle$ with $\left\langle u_{m} \mid u_{k}\right\rangle=\delta_{m k}$ and $\left|u_{k}\right\rangle \stackrel{\text { def }}{=} U|k\rangle$. Thus, from (50) and (51) it turns out that the Fisher information function remains unchanged under unitary operations. In the rest of the manuscript, we refer to Eq. (47) as the "parametric-independence constraint" on the Fisher information function.

As a side remark, we emphasize that within a statistical inference viewpoint extended to the quantum framework, the statistical notion of Fisher information function resembles a generalized mechanical notion of kinetic energy with respect to a given statistical parameter $(\theta$, in our case) regarded as temporal or spatial shift. Specifically, it can be shown that the following relation holds [40],

$$
\mathcal{K}(\theta)=\frac{1}{4} \mathcal{F}(\theta)+\sum_{k=0}^{N-1} J_{\theta}^{2}(k)\left|\psi_{\theta}(k)\right|^{2}
$$

with $\mathcal{F}(\theta)$ defined as in (50) and $\psi_{\theta}(k)=\left\langle k \mid \psi_{\theta}\right\rangle$ and $p_{k}(\theta)=\left|\psi_{\theta}(k)\right|^{2}$. The quantity $\mathcal{K}(\theta)$ denotes the kinetic energy of the wavefunction with respect to the parameter $\theta$ while $J_{\theta}(k)$ is a statistical analogue of the normalized quantum mechanical current density with respect to $\theta$. They are defined as [40],

$$
\mathcal{K}(\theta) \stackrel{\text { def }}{=} \sum_{k=0}^{N-1}\left|\frac{\partial \psi_{\theta}(k)}{\partial \theta}\right|^{2}
$$

and,

$$
J_{\theta}(k) \stackrel{\text { def }}{=} \frac{1}{2 i\left|\psi_{\theta}(k)\right|^{2}}\left(\frac{\partial \psi_{\theta}(k)}{\partial \theta} \psi_{\theta}^{*}(k)-\psi_{\theta}(k) \frac{\partial \psi_{\theta}^{*}(k)}{\partial \theta}\right),
$$

respectively. The symbol "*" denotes complex conjugation. For $\psi_{\theta}(k)=\sqrt{p_{k}(\theta)} e^{i \phi_{k}(\theta)}$, we obtain that

$$
\mathcal{K}(\theta)=\left\langle\dot{\psi}_{\theta} \mid \dot{\psi}_{\theta}\right\rangle, \mathcal{F}(\theta)=\sum_{k=0}^{N-1} \frac{\dot{p}_{k}^{2}}{p_{k}} \text { and, } J_{\theta}(k)=\dot{\phi}_{k}(\theta),
$$

with $\dot{\psi}_{\theta} \stackrel{\text { def }}{=} \frac{d \psi(\theta)}{d \theta}$. Therefore, Eq. (152) reads

$$
\left\langle\dot{\psi}_{\theta} \mid \dot{\psi}_{\theta}\right\rangle=\frac{1}{4} \sum_{k=0}^{N-1} \frac{\dot{p}_{k}^{2}}{p_{k}}+\sum_{k=0}^{N-1} p_{k} \dot{\phi}_{k}^{2}
$$

Since in Grover's case $\mathcal{F}(\theta)=4$ and $J_{\theta}(k)=0$ for any $k=0, \ldots, N-1$, condition (56) becomes

$$
\mathcal{K}(\theta)=\left\langle\dot{\psi}_{\theta} \mid \dot{\psi}_{\theta}\right\rangle=1
$$

We conclude that from a statistical quantum inference viewpoint, Grover's algorithm is characterized by a constant statistical kinetic energy where no "statistical dissipation" occurs.

The geodesic path related to Grover's algorithm is found by minimizing the action $\mathcal{S}\left[p_{k}(\theta)\right]$ defined as,

$$
\mathcal{S}\left[p_{k}(\theta)\right] \stackrel{\text { def }}{=} \int \sqrt{d s_{\mathrm{WY}}^{2}}=\int \mathcal{L}\left(\dot{p}_{k}(\theta), p_{k}(\theta)\right) d \theta
$$


where the Lagrangian-like quantity $\mathcal{L}\left(\dot{p}_{k}(\theta), p_{k}(\theta)\right)$ reads,

$$
\mathcal{L}\left(\dot{p}_{k}(\theta), p_{k}(\theta)\right)=\left[\sum_{k=0}^{N-1} \frac{\dot{p}_{k}^{2}(\theta)}{p_{k}(\theta)}\right]^{\frac{1}{2}},
$$

given the normalization constraint on the parametric probabilities $p_{k}(\theta)$,

$$
\sum_{k=0}^{N-1} p_{k}(\theta)=1
$$

For the sake of simplicity, consider the change of variable $p_{k}(\theta) \rightarrow q_{k}^{2}(\theta)$ [41]. Then, using the Lagrange multipliers method, the new action $\mathcal{S}^{\prime}\left[q_{k}(\theta)\right]$ to minimize becomes ,

$$
\mathcal{S}^{\prime}\left[q_{k}(\theta)\right]=\int \mathcal{L}^{\prime}\left(\dot{q}_{k}(\theta), q_{k}(\theta)\right) d \theta=\int\left\{\left[4 \sum_{k=1}^{N} \dot{q}_{k}^{2}(\theta)\right]^{\frac{1}{2}}-\lambda\left(\sum_{k=1}^{N} q_{k}^{2}(\theta)-1\right)\right\} d \theta,
$$

where $\lambda$ is the Lagrange multiplier and the new Lagrangian-like quantity is given by,

$$
\mathcal{L}^{\prime}\left(\dot{q}_{k}(\theta), q_{k}(\theta)\right)=\left[4 \sum_{k=1}^{N} \dot{q}_{k}^{2}(\theta)\right]^{\frac{1}{2}}-\lambda\left(\sum_{k=1}^{N} q_{k}^{2}(\theta)-1\right) .
$$

The path minimizing the action $\mathcal{S}^{\prime}\left[q_{k}(\theta)\right]$ satisfies the "actuality constraint",

$$
\frac{\delta \mathcal{S}^{\prime}\left[q_{k}(\theta)\right]}{\delta q_{k}(\theta)}=0
$$

leading to the Euler-Lagrange (EL) equation,

$$
\frac{d}{d \theta}\left(\frac{\partial \mathcal{L}^{\prime}\left(\dot{q}_{k}(\theta), q_{k}(\theta)\right)}{\partial \dot{q}_{k}}\right)-\frac{\partial \mathcal{L}^{\prime}\left(\dot{q}_{k}(\theta), q_{k}(\theta)\right)}{\partial q_{k}}=0
$$

From (62), it follows that

$$
\frac{d}{d \theta}\left(\frac{\partial \mathcal{L}^{\prime}}{\partial \dot{q}_{k}}\right)=\frac{4 \ddot{q}_{k}}{\left(4 \sum_{k=1}^{N} \dot{q}_{k}^{2}\right)^{\frac{1}{2}}}-\frac{\dot{q}_{k} \ddot{q}_{k}}{\left(4 \sum_{k=1}^{N} \dot{q}_{k}^{2}\right)^{\frac{1}{2}}} \frac{1}{\left(4 \sum_{k=1}^{N} \dot{q}_{k}^{2}\right)} 4 \dot{q}_{k}
$$

and,

$$
\frac{\partial \mathcal{L}^{\prime}}{\partial q_{k}}=-2 \lambda q_{k}
$$

Thus, substituting (66) and (65) into (64), the EL equation becomes

$$
\frac{d^{2} q_{k}(\theta)}{d \theta^{2}}-\frac{\dot{\mathcal{L}}\left(\dot{q}_{k}(\theta), q_{k}(\theta)\right)}{\mathcal{L}\left(\dot{q}_{k}(\theta), q_{k}(\theta)\right)} \frac{d q_{k}(\theta)}{d \theta}+\frac{\lambda}{2} \mathcal{L}\left(\dot{q}_{k}(\theta), q_{k}(\theta)\right) q_{k}(\theta)=0,
$$

where $\dot{\mathcal{L}}\left(\dot{q}_{k}(\theta), q_{k}(\theta)\right)=\frac{d \mathcal{L}\left(\dot{q}_{k}(\theta), q_{k}(\theta)\right)}{d \theta}$ with $\mathcal{L}\left(\dot{q}_{k}(\theta), q_{k}(\theta)\right)$ given in (59) with $q_{k}^{2}(\theta)=p_{k}(\theta)$. Using (47), it follows that $\mathcal{L}\left(\dot{q}_{k}(\theta), q_{k}(\theta)\right)$ is constant and equals two while $\dot{\mathcal{L}}\left(\dot{q}_{k}(\theta), q_{k}(\theta)\right)$ equals zero. It finally follows that

$$
q_{0}(\theta)=\sin \theta \text { and, } q_{\bar{k}}(\theta)=\frac{\cos \theta}{\sqrt{N-1}},
$$

where $\bar{k}=1, \ldots, N-1$ fulfil the Eq. (67) with $\lambda$ equal to one in order to satisfy the normalization condition (60). We then conclude that the $N$-dimensional probability vector $\vec{p} \equiv\left(p_{0}(\theta), p_{1}(\theta), \ldots, p_{N-1}(\theta)\right)$ with $p_{k}(\theta)$ defined in (31) is a geodesic path for which the quantum Fisher information action functional $\mathcal{S}^{\prime}\left[q_{k}(\theta)\right]$ achieves an extremal value. We also stress that such a path satisfies both the parametric-independence and actuality constraints in Eqs. (47) and (63), respectively. 


\section{DEVIATIONS FROM GROVER'S ALGORITHM}

As pointed out earlier, Grover's search algorithm relies on two key-assumptions: 1) Grover's iteration must be characterized only by means of unitary transformations acting on a uniform amplitude input state. This leads to the parametric-independence constraint on the Fisher information function (see Eq. (477)); 2) the expressions for the components of the probability distribution vectors associated to Grover's algorithm are such that the quantum Fisher information action functional is minimized when evaluated on such paths (see Eq. (63)). In summary, Grover's paths are actual unitary probability paths of constant Fisher information. When one of these two requirements (parametricinvariance and actuality) do not hold anymore, we are led to "deviations" from Grover's algorithm which should be somehow less efficient than Grover's one.

In what follows, we discuss unitary deviations from Grover's algorithm and attempt to quantify, whenever possible, different aspects from an information geometric point of view on manifolds where the statistical parameter $\theta$ can be regarded as a local coordinate parameterizing the density operator $\rho_{\theta}$.

\section{A. Grover's Model: Actuality and Parametric-Independence}

Recall that the probability distribution vectors associated with Grover's algorithm is given by,

$$
\vec{p}_{\mathrm{G}} \equiv\left(\sin ^{2} \theta, \frac{\cos ^{2} \theta}{N-1}, \ldots, \frac{\cos ^{2} \theta}{N-1}\right) .
$$

Eq. (69) leads to the constancy of the Fisher information function $\mathcal{F}(\theta)$,

$$
\mathcal{F}(\theta)=\sum_{k=0}^{N-1} \frac{1}{p_{k}}\left(\frac{d p_{k}(\theta)}{d \theta}\right)^{2}=4
$$

The geodesic equation in (21) for Grover's statistical model reads,

$$
\ddot{\theta}+\frac{1}{2 \mathcal{F}(\theta)} \frac{d \mathcal{F}(\theta)}{d \theta} \dot{\theta}^{2}=0
$$

with $\dot{\theta} \stackrel{\text { def }}{=} \frac{d \theta(\tau)}{d \tau}$. Since $\mathcal{F}(\theta)$ is constant, the geodesic equation reads $\ddot{\theta}=0$. Assuming that the boundary conditions are given by $\theta\left(\tau_{i}\right)=\theta_{i}$ and $\theta\left(\tau_{f}\right)=\theta_{f}$, integration of (71) leads to the geodesic path $\theta_{\mathrm{G}}(\tau)$,

$$
\theta_{\mathrm{G}}(\tau)=\frac{\theta_{i} \tau_{f}-\theta_{f} \tau_{i}}{\tau_{f}-\tau_{i}}+\left(\frac{\theta_{f}-\theta_{i}}{\tau_{f}-\tau_{i}}\right) \tau .
$$

The geodesic path $\theta_{\mathrm{G}}(\tau)$ can be regarded as a continuous succession of intermediate states connecting $\theta_{i}$ to $\theta_{f}$. The geodesic motion occurs with speed $v_{\mathrm{G}}(\tau)$,

$$
v_{\text {Grover }}(\tau) \stackrel{\text { def }}{=} \frac{d \theta_{\text {Grover }}(\tau)}{d \tau}
$$

satisfying the normalization relation,

$$
\left[g_{l m}(\theta) \frac{d \theta^{l}(\tau)}{d \tau} \frac{d \theta^{m}(\tau)}{d \tau}\right]^{\frac{1}{2}}=1,
$$

with $l=m=1$ and $g_{11}(\theta)=4$. Equation (174) implies that the temporal duration $\Delta \tau$ needed to navigate the geodesic path connecting $\theta_{i}$ to $\theta_{f}$ is given by,

$$
\Delta \tau \stackrel{\text { def }}{=} \tau_{f}-\tau_{i}=2\left(\theta_{f}-\theta_{i}\right) .
$$

We point out that $\Delta \tau$ can also be viewed as the length $L_{\mathrm{G}}\left(\theta_{i}, \theta_{f}\right)$ of the geodesic path connecting $\theta_{i}$ to $\theta_{f}$,

$$
L_{\mathrm{G}}\left(\theta_{i}, \theta_{f}\right) \stackrel{\text { def }}{=} \int_{\theta_{i}}^{\theta_{f}}\left[g_{l m}(\theta) d \theta^{l} d \theta^{m}\right]^{\frac{1}{2}}=\int_{\tau_{i}}^{\tau_{f}^{\prime}} d \tau\left[g_{l m}(\theta) \frac{d \theta^{l}(\tau)}{d \tau} \frac{d \theta^{m}(\tau)}{d \tau}\right]^{\frac{1}{2}} .
$$

Indeed, in the case being considered $L_{\mathrm{G}}\left(\theta_{i}, \theta_{f}\right)$ reads

$$
L_{\mathrm{G}}\left(\theta_{i}, \theta_{f}\right) \stackrel{\text { def }}{=} \int_{\theta_{i}}^{\theta_{f}} \sqrt{4} d \theta=2\left(\theta_{f}-\theta_{i}\right) \equiv \Delta \tau
$$




\section{B. Deviation from Grover's Model: Neither Actuality Nor Parametric-Independence}

In this example, we consider a properly normalized probability distribution vector given by,

$$
\vec{p}_{\text {Model-II }} \equiv\left(\theta^{2}, \frac{1-\theta^{2}}{N-1}, \ldots, \frac{1-\theta^{2}}{N-1}\right) .
$$

Notice that when $\theta \ll 1, \vec{p}_{\text {Grover }} \approx \vec{p}_{\text {Model-II }}$. The Fisher information function $\mathcal{F}(\theta)$ for the modified Grover's statistical model is not constant (no parametric-independence) and reads,

$$
\mathcal{F}(\theta)=\sum_{k=0}^{N-1} \frac{1}{p_{k}}\left(\frac{d p_{k}(\theta)}{d \theta}\right)^{2}=\frac{4}{1-\theta^{2}}
$$

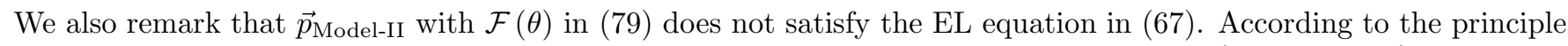
of least action, this means that $\vec{p}_{\text {Model-II }}$ can be regarded as a possible but not an actual (not extremal) probability path. Furthermore, the geodesic equation (21) for the modified Grover's statistical model becomes,

$$
\ddot{\theta}+\frac{\theta}{1-\theta^{2}} \dot{\theta}^{2}=0
$$

where $\dot{\theta}=\frac{d \theta(\tau)}{d \tau}$. Eq. (180) is a nonlinear second order ordinary differential equation. $\operatorname{Setting} \dot{\theta}=\frac{d \theta(\tau)}{d \tau}=y(\theta)$, Eq. (80) becomes a first order differential equation,

$$
y y^{\prime}+\frac{\theta}{1-\theta^{2}} y^{2}=0
$$

where $y^{\prime}=\frac{d y(\theta)}{d \theta}$. Assuming $y \neq 0$, integration of (81) implies that

$$
y(\theta)=C\left(1-\theta^{2}\right)^{\frac{1}{2}}
$$

where $C \in \mathbb{R}$ is an integration constant. Since $y(\theta)=\frac{d \theta(\tau)}{d \tau}$, it finally turns out that the general solution of Eq. (80) is given by,

$$
\theta(\tau)=\sin \left[C_{0}\left(\tau+C_{1}\right)\right]
$$

where $C_{0}$ and $C_{1}$ are real integration constants whose explicit expressions can be obtained once the boundary conditions are fixed. Assuming $\theta\left(\tau_{i}\right)=\theta_{i}$ and $\theta\left(\tau_{f}\right)=\theta_{f}$, the expression for the geodesic path $\theta_{\text {Model-II }}(\tau)$ reads,

$$
\theta_{\text {Model-II }}(\tau)=\sin \left[\left(\frac{\arcsin \theta_{f}-\arcsin \theta_{i}}{\tau_{f}^{\prime}-\tau_{i}^{\prime}}\right)\left(\frac{\tau_{f}^{\prime} \arcsin \theta_{i}-\tau_{i}^{\prime} \arcsin \theta_{f}}{\arcsin \theta_{f}-\arcsin \theta_{i}}+\tau\right)\right] .
$$

The geodesic path $\theta(\tau)$ can be regarded as a continuous succession of intermediate states connecting $\theta_{i}$ to $\theta_{f}$. The geodesic motion occurs with speed $v_{\text {Model-II }}(\tau)$,

$$
v_{\text {Model-II }}(\tau) \stackrel{\text { def }}{=} \frac{d \theta_{\text {Model-II }}(\tau)}{d \tau},
$$

satisfying the normalization relation,

$$
\left[g_{l m}(\theta) \frac{d \theta^{l}(\tau)}{d \tau} \frac{d \theta^{m}(\tau)}{d \tau}\right]^{\frac{1}{2}}=1
$$

with $l=m=1$ and $g_{11}(\theta)=4\left(1-\theta^{2}\right)^{-1}$. Equation (866) implies that the temporal duration $\Delta \tau^{\prime}$ needed to navigate the geodesic path connecting $\theta_{i}$ to $\theta_{f}$ is given by,

$$
\Delta \tau^{\prime} \stackrel{\text { def }}{=} \tau_{f}^{\prime}-\tau_{i}^{\prime}=2\left(\arcsin \theta_{f}-\arcsin \theta_{i}\right) .
$$


We point out that $\Delta \tau^{\prime}$ can also be viewed as the length $L\left(\theta_{i}, \theta_{f}\right)$ of the geodesic path connecting $\theta_{i}$ to $\theta_{f}$,

$$
L_{\text {Model-II }}\left(\theta_{i}, \theta_{f}\right) \stackrel{\text { def }}{=} \int_{\theta_{i}}^{\theta_{f}}\left[g_{l m}(\theta) d \theta^{l} d \theta^{m}\right]^{\frac{1}{2}}=\int_{\tau_{i}}^{\tau_{f}^{\prime}} d \tau\left[g_{l m}(\theta) \frac{d \theta^{l}(\tau)}{d \tau} \frac{d \theta^{m}(\tau)}{d \tau}\right]^{\frac{1}{2}} .
$$

Indeed, in the case being considered $L_{\text {Model-II }}\left(\theta_{i}, \theta_{f}\right)$ reads

$$
L_{\text {Model-II }}\left(\theta_{i}, \theta_{f}\right) \stackrel{\text { def }}{=} \int_{\theta_{i}}^{\theta_{f}} \sqrt{\frac{4}{1-\theta^{2}}} d \theta=2\left(\arcsin \theta_{f}-\arcsin \theta_{i}\right) \equiv \Delta \tau^{\prime} .
$$

Comparing Grover's model to the deviation from Grover's model and assuming $\theta_{f}=\theta_{i}+\epsilon$ with $\theta_{i} \equiv 0$ and $0<\epsilon \ll 1$, we obtain

$$
\Delta \tau_{\text {Model-II }}-\Delta \tau_{\text {Grover }} \simeq \frac{\epsilon^{3}}{3}+\mathcal{O}\left(\epsilon^{5}\right) \geq 0
$$

We may conclude that navigating along Grover's geodesic path is more efficient than navigating along the geodesic path characterizing the modified statistical model. Indeed, assuming that any navigation that is being compared occurs with the same unit speed, the efficiency of the navigation is simply quantified in terms of the length of the geodesic path connecting the initial and final states. Furthermore, since the length of a geodesic path can be regarded as the navigation duration, the most efficient unit-speed navigation is characterized by the shortest navigation duration (shortest geodesic path) for a given set of boundary conditions.

\section{Deviation from Grover's Model: Parametric-Independence without Actuality}

It is possible to consider normalized probability distribution vectors $\vec{p}_{\text {Model-III }} \equiv\left(p_{0}(\theta), p_{1}(\theta), \ldots, p_{N-1}(\theta)\right)$ that satisfy the parametric-independence constraint but do not minimize the action functional $\mathcal{S}^{\prime}\left[q_{k}(\theta)\right]$. For instance, assuming that $p_{k}(\theta)=p_{k^{\prime}}(\theta)$ for $k$ and $k^{\prime} \in\{1, \ldots, N-1\}$, the components of $\vec{p}_{\text {Model-III }}$ should satisfy the following system of two equations,

$$
p_{0}(\theta)+(N-1) p_{\bar{k}}(\theta)=1 \text { and, } \frac{\dot{p}_{0}^{2}(\theta)}{p_{0}(\theta)}+(N-1) \frac{\dot{p}_{\bar{k}}^{2}(\theta)}{p_{\bar{k}}(\theta)}=4,
$$

with $\bar{k}=1, \ldots, N-1$. From the first equation in (91), we get

$$
p_{\bar{k}}(\theta)=\frac{1-p_{0}(\theta)}{N-1}
$$

Substituting (92) into the second equation in (91), we obtain

$$
\dot{p}_{0}^{2}-4 p_{0}\left(1-p_{0}\right)=0 .
$$

Integrating (93) and using (92), a solution of the system (91) is given by,

$$
\vec{p}_{\text {Model-III }} \equiv\left(\frac{1+\sin 2 \theta}{2}, \frac{1-\sin 2 \theta}{2(N-1)}, \ldots, \frac{1-\sin 2 \theta}{2(N-1)}\right) .
$$

In this case, the Fisher information function $\mathcal{F}(\theta)=4$, however the components of $\vec{p}_{\text {Model-III }}$ do not satisfy the EL equation in (67). Although the integration of the geodesic equation in (21) for this model would lead to geodesic paths that are straight line trajectories, the components of $\vec{p}_{\text {Model-III }}$ represent only possible and not actual trajectories of the system (according to the principle of least action). Thus, the probability path $\vec{p}_{\text {Model-III }}$ lacks the actuality requirement that distinguishes Grover's probability path. Indeed, a part from an irrelevant constant factor, it turns out that the probability distribution vector related to Grover's algorithm is the only one satisfying both the parametricindependence and minimization condition (actuality constraint) simultaneously. 


\section{Deviation from Grover's Model: Actuality without Parametric-Independence}

For the sake of completeness, we mention one more possible scenario. There might exist a probability distribution vector $\vec{p}_{\text {Model-IV }} \equiv\left(p_{0}(\theta), p_{1}(\theta), \ldots, p_{N-1}(\theta)\right)$ with $p_{k}(\theta)=p_{k^{\prime}}(\theta)$ for $k$ and $k^{\prime} \in\{1, \ldots, N-1\}$ that satisfies the minimization without fulfilling the parametric-independence requirement. The components of such a path should satisfy the following system of nonlinear ordinary differential equations,

$$
\left\{\begin{array}{l}
\ddot{q}_{0}(\theta)-\frac{\dot{\mathcal{L}}(\theta)}{\mathcal{L}(\theta)} \dot{q}_{0}(\theta)+\frac{\mathcal{L}(\theta)}{2} q_{0}(\theta)=0, \\
\ddot{q}_{\bar{k}}(\theta)-\frac{\dot{\mathcal{L}}(\theta)}{\mathcal{L}(\theta)} \dot{q}_{\bar{k}}(\theta)+\frac{\mathcal{L}(\theta)}{2} q_{\bar{k}}(\theta)=0,
\end{array}\right.
$$

where $q_{0}^{2}(\theta)=p_{0}(\theta)$ and $q_{\bar{k}}^{2}(\theta)=p_{\bar{k}}(\theta)$ for $\bar{k}=1, \ldots, N-1$ with the normalization condition

$$
q_{0}^{2}(\theta)+(N-1) q_{\bar{k}}^{2}(\theta)=1
$$

The quantity $\mathcal{L}(\theta)$ in $(95)$ is defined as,

$$
\mathcal{L}(\theta) \stackrel{\text { def }}{=} 2\left[\dot{q}_{0}^{2}(\theta)+(N-1) \dot{q}_{\bar{k}}^{2}(\theta)\right]^{\frac{1}{2}} \neq \text { constant. }
$$

Using the normalization condition $(\underline{96})$, the expressions for $\mathcal{L}(\theta)$ and $\dot{\mathcal{L}}(\theta) \stackrel{\text { def }}{=} \frac{d \mathcal{L}(\theta)}{d \theta} \operatorname{read}$

$$
\mathcal{L}(\theta)=2 \dot{q}_{0} \sqrt{\frac{(N-1)\left(1-q_{0}^{2}\right)+q_{0}^{2}}{(N-1)\left(1-q_{0}^{2}\right)}}
$$

and

$$
\dot{\mathcal{L}}(\theta)=2 \ddot{q}_{0} \sqrt{\frac{(N-1)\left(1-q_{0}^{2}\right)+q_{0}^{2}}{(N-1)\left(1-q_{0}^{2}\right)}}+\frac{2 q_{0} \dot{q}_{0}^{2}}{(N-1)\left(1-q_{0}^{2}\right)^{2}} \sqrt{\frac{(N-1)\left(1-q_{0}^{2}\right)}{(N-1)\left(1-q_{0}^{2}\right)+q_{0}^{2}}},
$$

respectively. It is fairly clear that finding an analytical expression for actual $N$-dimensional $\vec{p}_{\text {Model-IV }}$ in the absence of parametric-independence may turn out to be quite nontrivial (for further details, see Appendix B). It is interesting to notice that the same computational challenge occurs when attempting to find analytical solutions of a non-unitary quantum walk 42]. However, focusing our attention on probability paths $\vec{p}$ with only two types of components, $p_{0}$ and $p_{\bar{k}}$ with $\bar{k}=1, \ldots, N-1$ and $N=2$, we are able to show that the only possible probability distribution vector satisfying the system (95) is Grover's path where $\mathcal{L}(\theta)$ is constant (and equals two). In such scenario, Eqs. (98) and (99) become

$$
\mathcal{L}(\theta)=\frac{2 \dot{q}_{0}}{\sqrt{1-q_{0}^{2}}} \text { and, } \dot{\mathcal{L}}(\theta)=\frac{2 \ddot{q}_{0}\left(1-q_{0}^{2}\right)+2 q_{0} \dot{q}_{0}^{2}}{\left(1-q_{0}^{2}\right)^{\frac{3}{2}}} .
$$

Using (100) together with the first equation of (95), we get

$$
\dot{q}_{0}-\sqrt{1-q_{0}^{2}}=0
$$

that is,

$$
q_{0}(\theta)=\sin \left(\theta+c_{0}\right) \text { and } q_{1}(\theta)=\cos \left(\theta+c_{0}\right)
$$

where $c_{0}$ is the real integration constant. We then conclude that in this special toy-case (one-qubit quantum Hilbert space, $\mathcal{H}_{2}^{1}$ with $\operatorname{dim}_{\mathbb{C}} \mathcal{H}_{2}^{1}=2=N$ ), actual paths are necessarily parametric-independent unitary paths as well. However, the realistic scenario implies a continuous approximation of Grover's quantum searching problem where $N \gg 1$. In such a case, actual parametric-dependent probability paths with the two-component structure may still exist (see Appendix B).

As a final remark, we point out that our information geometric analysis may be of use to investigate the possibility of the existence of actual parametric-dependent probability paths with a multi-component structure (more that two types of components). Such scenarios occur in a multi-item quantum search where more than a single item is being searched. 


\section{CONCLUDING REMARKS AND OPEN ISSUES}

In this article, we have quantified the notion of quantum distinguishability between parametric density operators by means of the Wigner-Yanase quantum information metric. We then presented an information geometric characterization of Grover's quantum search algorithm as a geodesic in the parameter space characterizing the pure state model, the manifold of the parametric density operators of pure quantum states constructed from the continuous approximation of the parametric quantum output state in Grover's algorithm. Finally, we discussed few possible deviations from Grover's algorithm within this quantum information geometric framework. For instance, in the second example in Section V, we have shown that navigating along Grover's geodesic path is more efficient than navigating along the geodesic path characterizing the modified statistical model. This statement holds when it is assumed that any navigation that is being compared occurs with the same unit speed and, therefore, the efficiency of the navigation is simply quantified in terms of the length of the geodesic path connecting the initial and final states. Furthermore, since the length of a geodesic path can be regarded as the navigation duration, we have also pointed out that the most efficient unit-speed navigation is characterized by the shortest navigation duration (shortest geodesic path) for a given set of boundary conditions.

The quantum information geometric techniques employed in our work were fairly simple. Unfortunately, we stress that there exist some technical difficulties in handling quantum information geometric techniques in general cases. For instance, there is no general formula for geodesic paths of manifolds of density operators endowed with an arbitrary given monotone Riemannian metric [43]. Explicit expressions are known only for two special metrics: the Bures metric [44] and the Wigner-Yanase metric [27]. In [44], geodesic paths on manifolds of density operators are obtained as projections of large circles on a large sphere within the purifying Hilbert-Schmidt space. In [27], geodesic paths are obtained exploiting the classical pull-back approach to Fisher information metric via sphere geometry. If diagonalization procedures are used, it is possible to obtain an explicit expression for the Bures distance of two arbitrary density matrices in the two-dimensional case [45]. In the general $n$-dimensional case this is unfortunately not possible. Thus, one of the greatest mathematical problems to face in order to quantify the metric properties of arbitrary mixed states is that of the explicit computation of eigenvalues of arbitrary Hermitian density matrices. The scenario becomes even more difficult if we recall that in order to find the geodesics one needs to integrate the geodesic equations expressed in terms of the metric and the Christoffel connection coefficients (which are expressed in terms of the first order changes in the metric). Furthermore, to integrate the Jacobi-Levi-Civita equation of geodesic spread, one also needs the explicit expression of the Riemannian curvature tensor (which encodes information about the second order changes in the metric tensor on the manifold of density operators). It may be that the first and second order perturbation theory of linear operators may turn out to be useful in estimating the changes in the metric tensor. However, it appears that thus far this line of investigation has not provided useful results [45]. There have been other attempts to compute in finite dimensions explicit formulae for the Bures metric where no diagonalization procedure is employed. These approaches only make use of the theory of matrix equations, determinants and traces [46]. Needless to say that further research is needed to fully handle the quantum information geometric formalism.

As pointed out in [1], quantum information geometry is only in its infancy, and much more research awaits to be performed. However, from the preliminary results obtained in this article, we have reason to think that quantum information geometric techniques may turn out to be especially useful for describing and understanding the efficiency of both unitary and non-unitary quantum walks used to implement quantum search algorithms in quantum computing [47]. In particular, our analysis opens up new lines of investigation that may deserve some attention. For instance, it would be worth deepening our analysis and attempting to understand the connection between computational complexity classes of quantum algorithms [48] and the complexity of the quantum geodesic paths associated with them. These investigations are left for future works.

\section{Acknowledgments}

C. C. thanks Marco Lucamarini and David Vitali for useful discussions. The research leading to these results has received funding from the European Commission's Seventh Framework Programme (FP7/2007-2013) under grant agreements no. 213681 .

\section{Appendix A: Derivation of the Wigner-Yanase line element}

Here we compute in an explicit manner the infinitesimal Wigner-Yanase line element $d s_{\mathrm{WY}}^{2}$,

$$
d s_{\mathrm{WY}}^{2}=4 d s_{\mathrm{FS}}^{2},
$$


where the Fubini-Study infinitesimal line element $d s_{\mathrm{FS}}^{2}$ is given by,

$$
d s_{\mathrm{FS}}^{2}=\|d \psi\|^{2}-|\langle\psi \mid d \psi\rangle|^{2}=1-\left|\left\langle\psi^{\prime} \mid \psi\right\rangle\right|^{2},
$$

where $|\psi\rangle$ and $\left|\psi^{\prime}\right\rangle$ are neighboring normalized pure states expanded in an orthonormal basis $\{|k\rangle\}$ with $k \in\{1, \ldots, N\}$,

$$
|\psi\rangle=\sum_{k=1}^{N} \sqrt{p_{k}(\theta)} e^{i \phi_{k}(\theta)}|k\rangle \text { and, }\left|\psi^{\prime}\right\rangle=\sum_{k=1}^{N} \sqrt{p_{k}+d p_{k}} e^{i\left(\phi_{k}+d \phi_{k}\right)}|k\rangle,
$$

respectively. Observe that,

$$
\begin{aligned}
\left|\psi^{\prime}\right\rangle & =\sum_{k=1}^{N} \sqrt{p_{k}+d p_{k}} e^{i\left(\phi_{k}+d \phi_{k}\right)}|k\rangle=\sum_{k=1}^{N}\left[\sqrt{p_{k}} \sqrt{1+\frac{d p_{k}}{p_{k}}} e^{i \phi_{k}}\left(1+i d \phi_{k}+\frac{1}{2}\left(i d \phi_{k}\right)^{2}\right)\right]|k\rangle \\
& =\sum_{k=1}^{N}\left[\sqrt{p_{k}}\left(1+\frac{1}{2} \frac{d p_{k}}{p_{k}}-\frac{1}{8} \frac{d p_{k}^{2}}{p_{k}^{2}}\right) e^{i \phi_{k}}\left(1+i d \phi_{k}-\frac{1}{2} d \phi_{k}^{2}\right)\right]|k\rangle .
\end{aligned}
$$

Eq. (A4) implies that,

$$
\left\langle\psi^{\prime}\right|=\sum_{k=1}^{N}\left[\sqrt{p_{k}}\left(1+\frac{1}{2} \frac{d p_{k}}{p_{k}}-\frac{1}{8} \frac{d p_{k}^{2}}{p_{k}^{2}}\right) e^{-i \phi_{k}}\left(1-i d \phi_{k}-\frac{1}{2} d \phi_{k}^{2}\right)\right]\langle k|,
$$

and thus $\left\langle\psi^{\prime} \mid \psi\right\rangle$ becomes,

$$
\begin{aligned}
\left\langle\psi^{\prime} \mid \psi\right\rangle & =\sum_{k=1}^{N}\left[p_{k}\left(1+\frac{1}{2} \frac{d p_{k}}{p_{k}}-\frac{1}{8} \frac{d p_{k}^{2}}{p_{k}^{2}}\right)\left(1-i d \phi_{k}-\frac{1}{2} d \phi_{k}^{2}\right)\right] \\
& =\sum_{k=1}^{N}\left[\left(p_{k}+\frac{1}{2} d p_{k}-\frac{1}{8} \frac{d p_{k}^{2}}{p_{k}}\right)\left(1-i d \phi_{k}-\frac{1}{2} d \phi_{k}^{2}\right)\right] \\
& =\sum_{k=1}^{N}\left(p_{k}+\frac{1}{2} d p_{k}-\frac{1}{8} \frac{d p_{k}^{2}}{p_{k}}\right)-i \sum_{k=1}^{N}\left(p_{k}+\frac{1}{2} d p_{k}-\frac{1}{8} \frac{d p_{k}^{2}}{p_{k}}\right) d \phi_{k}-\frac{1}{2} \sum_{k=1}^{N} p_{k} d \phi_{k}^{2} \\
& =1-\frac{1}{8} \sum_{k=1}^{N} \frac{d p_{k}^{2}}{p_{k}}-i \sum_{k=1}^{N} p_{k} d \phi_{k}-\frac{i}{2} \sum_{k=1}^{N} d p_{k} d \phi_{k}-\frac{1}{2} \sum_{k=1}^{N} p_{k} d \phi_{k}^{2},
\end{aligned}
$$

where we have made use of the normalization constraint and its differential form,

$$
\sum_{k=1}^{N} p_{k}=1 \text { and, } \sum_{k=1}^{N} d p_{k}=0
$$


respectively. We then obtain that $\left|\left\langle\psi^{\prime} \mid \psi\right\rangle\right|^{2}$ reads,

$$
\begin{aligned}
\left|\left\langle\psi^{\prime} \mid \psi\right\rangle\right|^{2}= & \left\langle\psi^{\prime} \mid \psi\right\rangle\left\langle\psi^{\prime} \mid \psi\right\rangle^{*} \\
= & {\left[1-\frac{1}{8} \sum_{k=1}^{N} \frac{d p_{k}^{2}}{p_{k}}-i \sum_{k=1}^{N} p_{k} d \phi_{k}-\frac{i}{2} \sum_{k=1}^{N} d p_{k} d \phi_{k}-\frac{1}{2} \sum_{k=1}^{N} p_{k} d \phi_{k}^{2}\right] . } \\
& \cdot\left[1-\frac{1}{8} \sum_{k=1}^{N} \frac{d p_{k}^{2}}{p_{k}}+i \sum_{k=1}^{N} p_{k} d \phi_{k}+\frac{i}{2} \sum_{k=1}^{N} d p_{k} d \phi_{k}-\frac{1}{2} \sum_{k=1}^{N} p_{k} d \phi_{k}^{2}\right] \\
= & 1-\frac{1}{8} \sum_{k=1}^{N} \frac{d p_{k}^{2}}{p_{k}}+i \sum_{k=1}^{N} p_{k} d \phi_{k}+\frac{i}{2} \sum_{k=1}^{N} d p_{k} d \phi_{k}-\frac{1}{2} \sum_{k=1}^{N} p_{k} d \phi_{k}^{2}-\frac{1}{8} \sum_{k=1}^{N} \frac{d p_{k}^{2}}{p_{k}}+ \\
& -i \sum_{k=1}^{N} p_{k} d \phi_{k}+\left(\sum_{k=1}^{N} p_{k} d \phi_{k}\right)^{2}-\frac{i}{2} \sum_{k=1}^{N} d p_{k} d \phi_{k}-\frac{1}{2} \sum_{k=1}^{N} p_{k} d \phi_{k}^{2} \\
= & 1-\frac{1}{4} \sum_{k=1}^{N} \frac{d p_{k}^{2}}{p_{k}}-\sum_{k=1}^{N} p_{k} d \phi_{k}^{2}+\left(\sum_{k=1}^{N} p_{k} d \phi_{k}\right)^{2} \cdot
\end{aligned}
$$

In conclusion, the infinitesimal Wigner-Yanase line element $d s_{\mathrm{WY}}^{2}$ becomes

$$
\begin{aligned}
d s_{\mathrm{WY}}^{2} & =4\left\{\frac{1}{4} \sum_{k=1}^{N} \frac{d p_{k}^{2}}{p_{k}}+\left[\sum_{k=1}^{N} p_{k} d \phi_{k}^{2}-\left(\sum_{k=1}^{N} p_{k} d \phi_{k}\right)^{2}\right]\right\} \\
& =\sum_{k=1}^{N} \frac{d p_{k}^{2}}{p_{k}}+4\left[\sum_{k=1}^{N} p_{k} d \phi_{k}^{2}-\left(\sum_{k=1}^{N} p_{k} d \phi_{k}\right)^{2}\right] \\
& =\left\{\sum_{k=1}^{N} \frac{1}{p_{k}}\left(\frac{d p_{k}}{d \theta}\right)^{2}+4\left[\sum_{k=1}^{N} p_{k}\left(\frac{d \phi_{k}}{d \theta}\right)^{2}-\left(\sum_{k=1}^{N} p_{k} \frac{d \phi_{k}}{d \theta}\right)^{2}\right]\right\} d \theta^{2} \\
& =\left\{\sum_{k=1}^{N} \frac{\dot{p}_{k}^{2}}{p_{k}}+4\left[\sum_{k=1}^{N} p_{k} \dot{\phi}_{k}^{2}-\left(\sum_{k=1}^{N} p_{k} \dot{\phi}_{k}\right)^{2}\right]\right\} d \theta^{2},
\end{aligned}
$$

that is,

$$
d s_{\mathrm{WY}}^{2}=\left\{\sum_{k=1}^{N} \frac{\dot{p}_{k}^{2}}{p_{k}}+4\left[\sum_{k=1}^{N} p_{k} \dot{\phi}_{k}^{2}-\left(\sum_{k=1}^{N} p_{k} \dot{\phi}_{k}\right)^{2}\right]\right\} d \theta^{2}
$$

where,

$$
\dot{p}_{k}=\frac{d p_{k}(\theta)}{d \theta} \text { and, } \dot{\phi}_{k}=\frac{d \phi_{k}(\theta)}{d \theta}
$$

\section{Appendix B: Actual Parametric-Dependent Unitary Probability Distribution Vectors}

Substituting (98) and (99) into the first equation in (95), the EL equation reads

$$
\frac{d q_{0}(\theta)}{d \theta}-\frac{1}{\sqrt{N-1}}\left(1-q_{0}^{2}\right)^{\frac{1}{2}}\left[(N-1)\left(1-q_{0}^{2}\right)+q_{0}^{2}\right]^{\frac{3}{2}}=0,
$$


that is,

$$
\mathcal{I}_{N}\left(q_{0}(\theta)\right) \stackrel{\text { def }}{=} \int \frac{d q_{0}}{\left(1-q_{0}^{2}\right)^{\frac{1}{2}}\left[(N-1)\left(1-q_{0}^{2}\right)+q_{0}^{2}\right]^{\frac{3}{2}}}=\frac{1}{\sqrt{N-1}} \int d \theta .
$$

The integral $\mathcal{I}\left(q_{0}(\theta)\right)$ on the left-hand side of (B2) can be expressed in terms of an elliptic integral of the second kind $E[\cdot \mid \cdot]$ (see [49], for instance) as follows,

$$
\mathcal{I}_{N}\left(q_{0}(\theta)\right)=\frac{\sqrt{N\left(1-q_{0}^{2}\right)+2 q_{0}^{2}-1}}{(N-1)} \cdot\left[\frac{(N-2) q_{0} \sqrt{1-q_{0}^{2}}}{N\left(q_{0}^{2}-1\right)-2 q_{0}^{2}+1}+\frac{E\left(\sin ^{-1}\left(q_{0}\right) \mid \frac{N-2}{N-1}\right)}{\sqrt{\frac{N\left(1-q_{0}^{2}\right)+2 q_{0}^{2}-1}{N-1}}}\right] .
$$

Finding an analytical solution $q_{0}(\theta)$ satisfying the following equation,

$$
\mathcal{I}_{N}\left(q_{0}(\theta)\right)-\left[\frac{1}{\sqrt{N-1}} \theta+C_{N}\right]=0
$$

where $C_{N}$ is a real integration constant is quite challenging. Approximate solutions to Eq. (B4) would require numerical investigations which are left for elsewhere. However, in the two-dimensional case $(N=2)$, we get a closed form solution for $q_{0}(\theta)$. In such a case $\mathcal{I}_{2}\left(q_{0}(\theta)\right)$ reads,

$$
\mathcal{I}_{2}\left(q_{0}(\theta)\right)=E\left(\sin ^{-1}\left(q_{0}\right) \mid 0\right) \stackrel{\text { def }}{=} \arcsin \left(q_{0}(\theta)\right),
$$

that is,

$$
q_{0}(\theta)=\sin \left(\theta+C_{2}\right)
$$

As a conclusive remark, we stress that although finding an analytical solution $q_{0}(\theta)$ for Eq. (B4) may not be trivial, for sure $q_{0}(\theta)$ in $(\underline{\mathrm{B} 6})$ does not satisfy this equation when $N \neq 2$. This leaves open the possibility of finding actual parametric-dependent unitary probability paths also in the case of a single-item quantum search.

[1] S. Amari and H. Nagaoka, "Methods of Information Geometry", Oxford University Press (2000).

[2] W. Janke et al., "Information geometry of the spherical model", Phys. Rev. E67, 046106 (2003).

[3] W. Janke et al., "Information geometry and phase transitions", Physica A336, 181 (2004).

[4] W. Janke et al., "Information geometry of the Ising model on planar random graphs", Phys. Rev. E66, 056119 (2002).

[5] B. P. Dolan et al., "The information geometry of the one-dimensional Potts model", J. Phys. A: Math. Gen. 35, 9025 (2002).

[6] S. L. Braunstein and C. M. Caves, "Statistical Distance and the Geometry of Quantum States", Phys. Rev. Lett. 72, 3439 (1994).

[7] C. Cafaro, "Information-geometric indicators of chaos in Gaussian models on statistical manifolds of negative Ricci curvature", Int. J. Theor. Phys. 47, 2924 (2008).

[8] C. Cafaro, "Information geometry, inference methods and chaotic energy levels statistics", Mod. Phys. Lett. B22, 1879 (2008).

[9] C. Cafaro and S. A. Ali, "Can chaotic quantum energy levels statistics be characterized using information geometry and inference methods?", Physica A387, 6876 (2008).

[10] C. Cafaro and S. Mancini, "Quantifying The Complexity Of Geodesic Paths On Curved Statistical Manifolds Through Information Geometric Entropies and Jacobi Fields", Physica D240, 607 (2011).

[11] J. Summhammer, "Maximum predictive power and the superposition principle", Int. J. Theor. Phys. 33, 171 (1994).

[12] J. Wheeler, in Complexity, Entropy, and Physics of Information, edited by Z. H. Zurek, Addison-Wesley, pp.3-28 (1990).

[13] A. Caticha and C. Cafaro, "From information geometry to Newtonian dynamics", in Bayesian Inference and Maximum Entropy Methods in Science and Engineering, AIP Conf. Proc. 954, 165 (2007).

[14] P. Goyal, "From Information Geometry to Quantum Theory", New. J. Phys. 12, 023012 (2010).

[15] A. Caticha, "From Entropic Dynamics to Quantum Theory", in Bayesian Inference and Maximum Entropy Methods in Science and Engineering, AIP Conf. Proc. 1193, 48 (2009).

[16] M. A. Nielsen et. al., "Quantum Computation as Geometry", Science 311, 1133 (2006).

[17] M. R. Dowling and M. A. Nielsen, "The Geometry of Quantum Computation", Quantum Information \& Computation 8, 0861 (2008). 
[18] H. E. Brandt, "Riemannian curvature in the differential geometry of quantum computation", Physica E42, 449 (2010).

[19] L. K. Grover, "Quantum Mechanics Helps in Searching for a Needle in a Haystack", Phys. Rev. Lett. 79, 325 (1997).

[20] A. Miyake and M. Wadati, "Geometric strategy for the optimal quantum search", Phys. Rev. A64, 042317 (2001).

[21] J. J. Alvarez and C. Gomez, "A Comment on Fisher Information and Quantum Algorithms", arXiv:quant-ph/9910115 (2000).

[22] N. N. Cencov, "Statistical decision rules and optimal inference", Transl. Math. Monographs, vol. 53, Amer. Math. Soc., Providence-RI (1981).

[23] L. L. Campbell, "An extended Cencov characterization of the information metric", Proc. Am. Math. Soc. 98, 135 (1986).

[24] E. A. Morozova and N. N. Cencov, "Markov Invariant Geometry on State Manifolds" (in Russian), Itogi Nauki i Tekhniki 36, 69 (1990).

[25] D. Petz, "Monotone Metrics on Matrix Spaces", Lin. Alg. Appl. 244, 81 (1996).

[26] M. R. Grasselli and R. F. Streater, "On the uniqueness of the Chentsov metric in quantum information geometry", InfiniteDimensional Anal. Quantum Prob. 4, 173 (2001).

[27] P. Gibilisco and S. Isola, "Wigner-Yanase information on quantum state space: the geometric approach", J. Math. Phys. 44, $3752(2003)$.

[28] E. P. Wigner and M. Yanase, "Information content of distribution", Proc. Nat. Acad. Sci. USA 49, 910 (1963).

[29] S. Luo, "Fisher Information for Wavefunctions: Classical and Quantum", Chin. Phys. Lett. 23, 3127 (2006).

[30] L. D. Landau and E. M. Lifshitz, "The Classical Theory of Fields", Pergamon, London (1962).

[31] N. D. Mermin, "Quantum Computer Science: An Introduction", Cambridge University Press (2007).

[32] C. H. Bennett et al., "Strengths and Weaknesses of Quantum Computing", SIAM J. Comput. 26, 1510 (1997).

[33] L. K. Grover, "A fast quantum mechanical algorithm for database search", Proceedings of the 28th Annual ACM Symposium on Theory of Computing, Philadelphia USA, pp. 212-219 (1996)

[34] M. Boyer et al., "Tight bounds on quantum searching", Proceedings of the Fourth Workshop on Physics and Computation, Boston USA, New England Complex Systems Institute, pp. 36-43 (1996).

[35] C. Zalka, " Grover's quantum searching algorithm is optimal", Phys. Rev. A60, 2746 (1999).

[36] P. W. Shor, "Polynomial-time algorithms for prime factorization and discrete logarithms on a quantum computer", SIAM J. Sci. Statist. Comput. 26, 1484 (1997).

[37] J. P. Provost and G. Vallee, "Riemannian Structure on Manifolds of Quantum States", Comm. Math. Phys. 76, 289 (1980).

[38] A. Fujiwara and H. Nagaoka, "Quantum Fisher metric and estimation for pure state models", Phys. Lett. A201, 119 (1995).

[39] S. Brandt, "Statistical and Computational Methods in Data Analysis", North Holland Publishing Company (1970).

[40] S. Luo, "Fisher information, kinetic energy and uncertainty relation inequalities", J. Phys. A: Math. Gen. 35, 5181 (2002).

[41] W. K. Wootters, "Statistical distance and Hilbert space", Phys. Rev. D23, 357 (1981).

[42] V. Kendon and O. Maloyer, "Optimal computation with non-unitary quantum walks", Theoretical Computer Science 394, 187 (2008).

[43] A. Cencova, "Geodesic distances on density matrices", J. Math. Phys. 45, 1787 (2004).

[44] J. Dittmann and A. Uhlmann, "Connections and metrics respecting standard purification", J. Math. Phys. 40, 3246 (1999).

[45] M. Hubner, "Explicit computation of the Bures distance for density matrices", Phys. Lett. A163, 239 (1992).

[46] J. Dittmann, "Explicit formulae for the Bures metric", J. Phys. A32, 2663 (1999).

[47] N. Shenvi et al., "A Quantum Random Walk Search Algorithm", Phys. Rev. A67, 052307 (2003).

[48] M. A. Nielsen and I. L. Chuang, "Quantum Computation and Information", Cambridge University Press (2000).

[49] M. Abramowtz and I. Stegun, "Handbook of Mathematical Functions: with Formulas, Graphs, and Mathematical Tables", Dover Publications, INC., New York (1972). 